\title{
APPLICATION OF NMR TECHNIQUE IN THE ELUCIDATION OF MARINE NATURAL COMPOUNDS
}

\author{
Maria Letizia Ciavatta \\ Istituto di Chimica Biomolecolare, (CNR), Via Campi Flegrei $34-I-80078$ Pozzuoli \\ e-mail: lciavatta@icb.cnrit; Phone00390818675243-Fax00390818675340
}

\begin{abstract}
The topic of the seminar held in the Institute of Chemistry, Academy of Sciences of Moldova on $30^{\text {th }}$ September in the frame of the joint Moldo-Italian seminar "New frontiers in natural product chemistry", concerned the use of NMR techniques in the elucidation of natural products. Step by step, two marine compounds (Fulvyne C and Tritoniopsin A) belonging to different chemical classes have been analyzed, by using suitable NMR experiments. This powerful technique allowed the elucidation of compounds as fulvynes, long chain polyacetylenes with the same functional groups but differently located in the chain, as well as tritoniopsins, cyclic diterpenes with a new skeleton, providing further information on their relative and absolute stereochemistry.
\end{abstract}

\section{Introduction}

Nuclear Magnetic Resonance spectroscopy [1] has become the most appreciated technique by the natural product chemists letting to achieve the chemical structures, even complicated and in little amount, of compounds isolated from different sources. Together with Mass Spectrometry, this technique aided us to establish the structures of nine long chain oxygenated polyacetylenes [2] from the Mediterranean sponge Haliclona fulva (Case 1) and to describe four cladiellane based diterpenes [3] from the nudibranch mollusc Tritoniopsis elegans (Case 2) collected in the South China Sea.

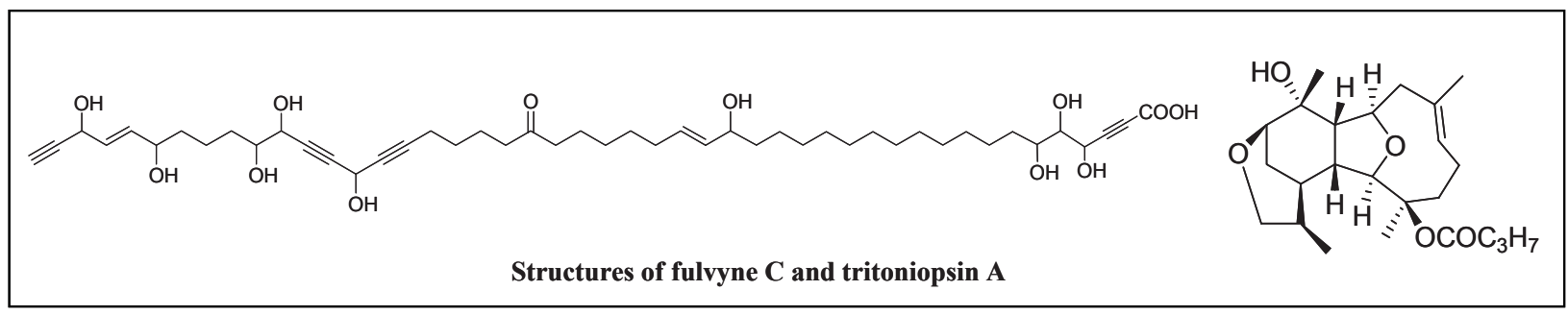

\section{The first step}

Once obtained a pure compound by using chromatographic means we have to calculate the molecular formula from the high-resolution mass spectrometry (HRMS): it will give the weight and molecular formula of our compounds and information about the presence of unsaturation degrees given by double and triple bonds and eventually by cyclic rings.

\section{The NMR experiments}

First, the analysis of the proton spectra of unknown compounds, recorded in a suitable deuterated solvent, can give a series of useful information: a) signals absorbing at different chemical shifts correspond to protons of different functional groups; b) the intensities of the signals are directly proportional to the number of protons generating them; c) the shape of signals (multiplets) gives information about the environment of a given nucleus through the analysis of their coupling constant. Second, the ${ }^{13} \mathrm{C}$ NMR experiments show how many carbons the molecules under investigation contain and which functional groups are present. We can use the ${ }^{13} \mathrm{C}$-DEPT sequence to obtain important information about the number of $\mathrm{CH}_{3}, \mathrm{CH}_{2}$ and $\mathrm{CH}$ and by difference with the carbon experiment the number of quaternary carbons.

To connect each carbon resonance with the proton directly linked, the HSQC bidimensional experiment has to be performed. The analysis of hetero-nuclear correlation spectra is carried out simply by tracing the coordinates of each cross-peaks with both the proton and the carbon chemical shift scales. Thus a list of correlation between protonated carbons and the attached protons is constructed.

The next step is to carry out a COSY experiment in which you can observe the scalar coupling and so connect geminal and vicinal protons. This experiment appears as a contour plot map with a square diagram symmetric with respect to a diagonal from lower left to upper right. Coupled protons are recognized by an off-diagonal peak at the coordinated corresponding to the chemical shift of the two coupled protons. During the analysis of a COSY spectrum you can draw some partial structures of the compound under investigation. For example in Case 1, these experiments led us to construct the fragments a-e of fulvyne $\mathrm{C}$, as drawn below. 


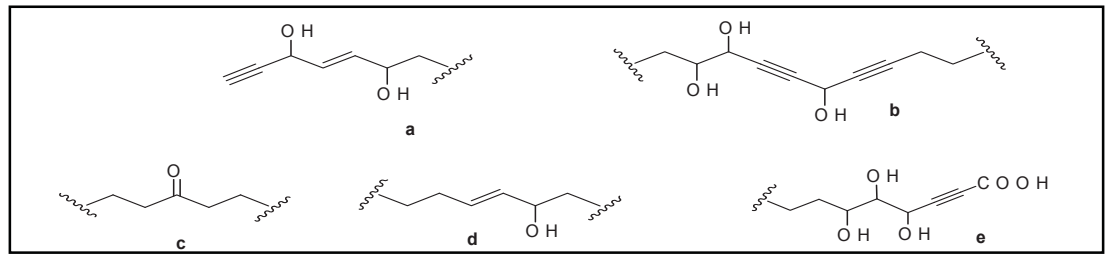

Partial structures deduced by COSY

The HMBC experiment finally gives useful information about the carbons and the protons not directly linked, but 2-4 bonds far. Careful analysis of HMBC spectrum usually allows the connection of the different spin systems identified by COSY correlations whose carbon counterpart has been connected by HSQC.

\section{Further NMR experiments}

Sometimes these fundamental NMR sequences are not sufficient to establish unambiguously the chemical structures of new compounds. As example, fulvyne C shows NMR spectra very similar to those of another co-occurring polyacetylene (fulvyne B), therefore they have to possess the same functional groups but located in different fashion along the chain. Analysis of TOCSY experiments recorded on both fulvynes allows to discriminate the two different sequences by defining all proton correlations in a mutually coupled spin system and consequently by connecting the partial structures detected from COSY.
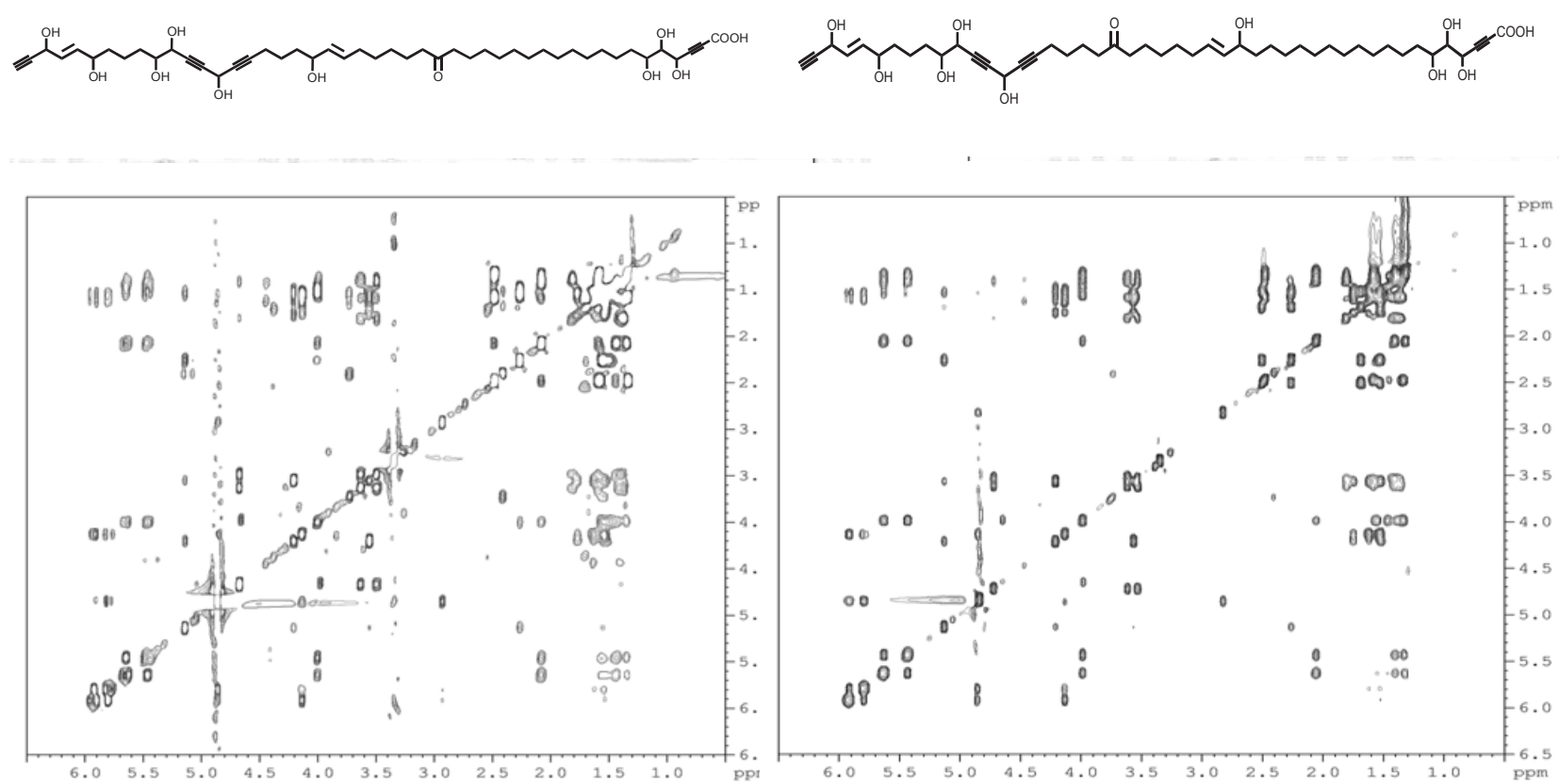

TOCSY spectra for fulvyne C (left) and B (right)

In the second case (tritoniopsin A), once obtained the molecular formula and the number of unsaturation degrees from the HRMS we have carried out the mono-dimensional NMR experiments. From the proton and carbon spectra we immediately get information about the nature of the compound under investigation. Indeed, tritoniopsin A shows a typical terpene pattern due to the presence of several methyls. ${ }^{13} \mathrm{C}-\mathrm{NMR}$ and DEPT spectra of tritoniopsin A show 24 carbon resonances (as indicated by the molecular formula), five of which are methyl groups, seven are methylenes, eight are methines and the remaining four are quaternary carbons.

Even in this case, information about which carbons and protons are directly bonded can be obtained by recording the direct hetero-correlation experiment (HSQC).

The spin systems present in the molecule can be deduced from COSY experiment, as shown below, whereas the analysis of HMBC correlations can aid us to connect the corresponding partial structures (main long-range cross-peaks are represented by arrows) and to construct the structure. 

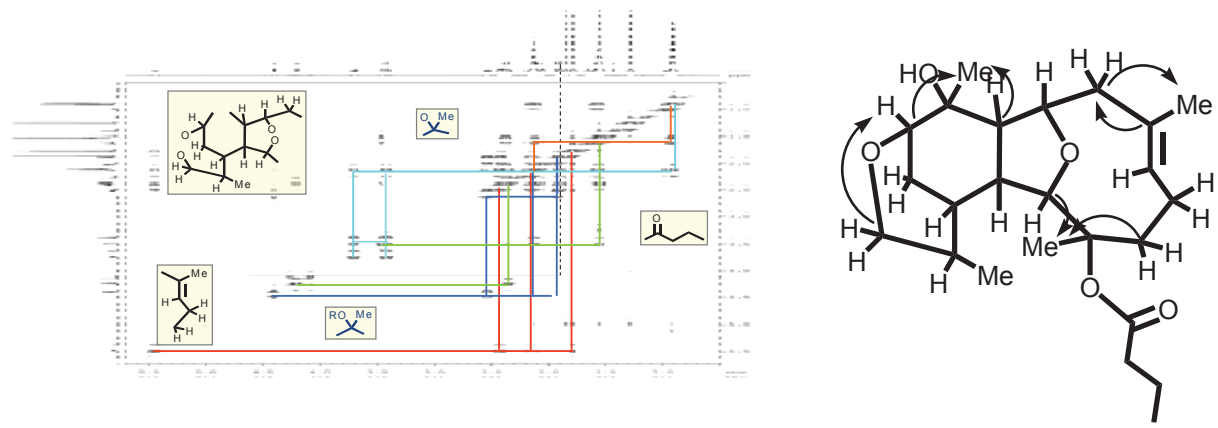

Due to the presence of nine stereogenic centers in tritoniopsin A, the objective is to give correct information on their relative configuration. Some of these can be easily deduced from the analysis of the proton spectrum signals as well as from the carbon chemical shift value. In tritoniopsin A because of the presence of a cyclohexane ring, we can suggest the configuration at two carbons by the shape of the corresponding protons (H-12 and H-14 are equatorially oriented because appear as broad singlets). Moreover, the carbon value of the methyl attached to $\mathrm{C}-11$ on the same ring is consistent with the equatorial position. To confirm these evidences and to establish the configuration of the remaining carbons, mono- and bi-dimensional experiments (nOe diff and NOESY) based on dipole-dipole mechanism (two nuclei not directly linked but spatially close can mutually interact through space) can be carried out obtaining very indicative information.
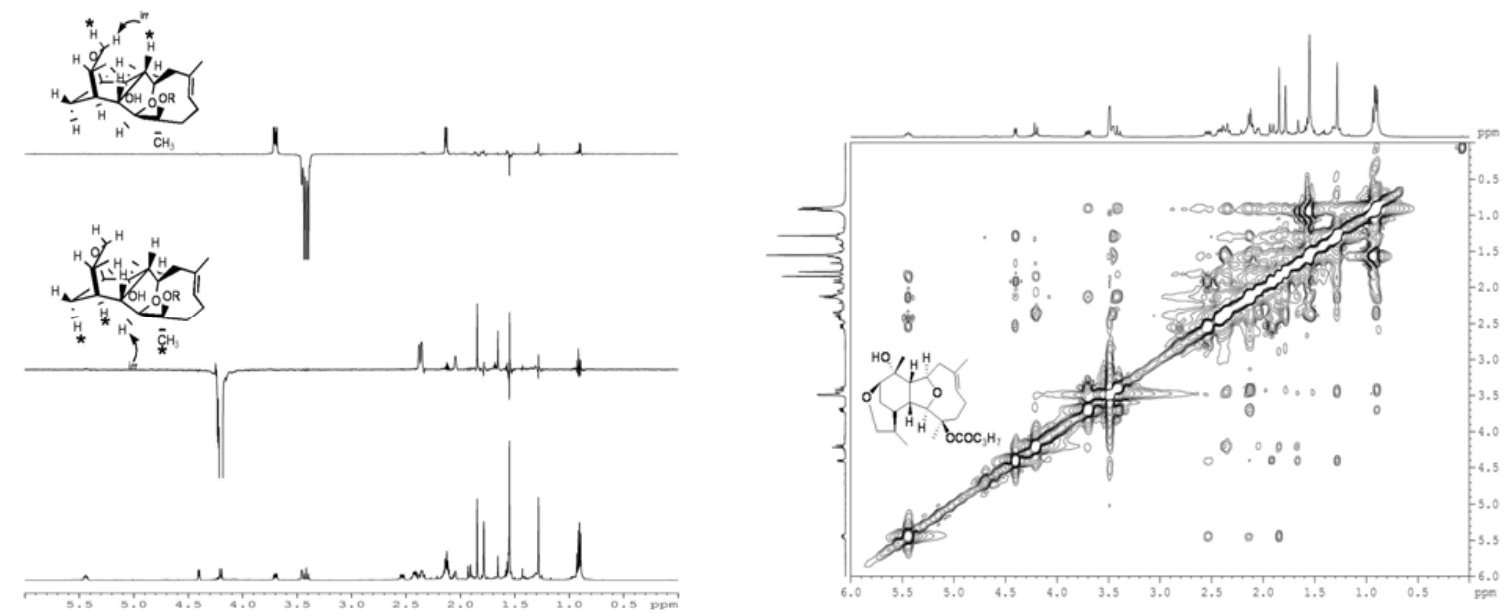

Another example of the versatile use of NMR technique is represented by the application of the Mosher method for the determination of the absolute stereochemistry of tritoniopsins. The presence of a secondary alcohol in one of the isolated tritoniopsins makes this molecule a useful model for the application of the method [4,5] leading to the assignment of the absolute stereochemistry at the carbinol center. The method is based on the conversion of the alcohol into two diastereomeric esters displaying different ${ }^{1} \mathrm{H}-\mathrm{NMR}$ spectra. The evaluation of the difference in the chemical shift values of protons in the two esters $(\Delta \delta S-\Delta \delta R)$ allows the absolute stereochemistry to be established as depicted below.

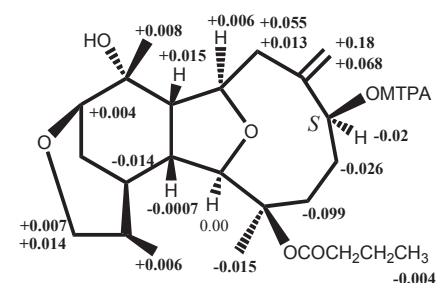

\section{Conclusions}

Nuclear Magnetic Resonance techniques represent a powerful tool for the structural determination of organic compounds, particularly in the field of natural products. It requires only minute amount of substance and frequently allows the determination of stereochemical details or even preferred conformation in solution. Nowadays, the variety of experiments available on modern NMR instruments has reduced the time needed for the acquisition data. 
Acknowledments. Academy of Sciences (Moldova) and CNR (Italy) are acknowledged for financial support (bilateral project "Novel approaches for the synthesis of optically active cannabinoids with relevant biological activity and therapeutical potential" 2011-2012).

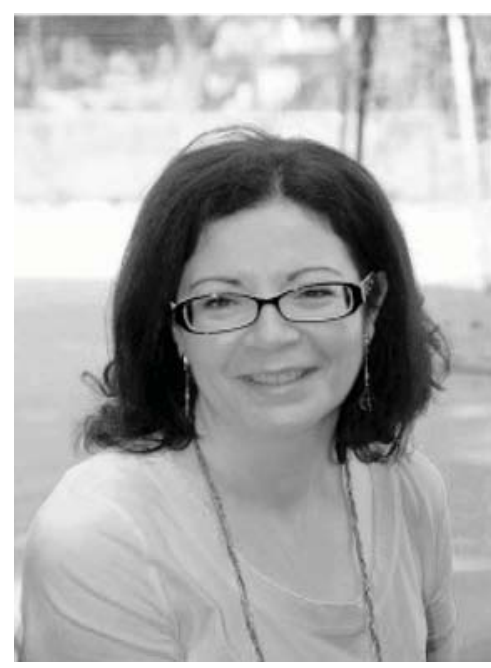

Dr. Maria Letizia Ciavatta was born in 1965 in Naples. She graduated in Pharmacy from University of Naples "Federico II" in 1988 under the supervision of Prof. E. Fattorusso. In 1991 she obtained a master in Officinal Plant Science from University of Naples. After she moved to CNR as a fellow with Professor G. Cimino (ICMIB, Naples, Italy), where she got her training first on some derivatives of cholesterol in food matrix and then on marine natural products. From 1999 she is a researcher of the laboratory of marine natural chemistry, at the Institute of Biomolecular Chemistry, CNR, Italy. Her research interests include the chemical analysis and separation of complex mixtures of natural products of marine, plant and microbial origin with particular attention to structural characterization (NMR and mass) of molecules that show activity as lead-compounds in pharmaceutical field. She is author of more than 60 scientific papers.

\section{References}

[1]. For interpretation of basic NMR experiments consult "Spectrometric Identification of Organic Compounds" Silverstein, R.M.; Webster, F.X.; Kiemle, D. Wiley \& Sons Inc. Ed., 2005, U.S.

[2]. Nuzzo, G.; Ciavatta, M.L.; Villani, G.; Manzo, E.; Zanfardino, A.; Varcamonti, M.; Gavagnin, M. Tetrahedron., 2012, 68, 750-760, in press.

[3]. Ciavatta, M.L.; Manzo, E.; Mollo, E.; Mattia, C.A.; Tedesco, C.; Irace, C.; Guo, Y.W.; Li, X.B.; Cimino, G.; Gavagnin, M. J. Nat. Prod., 2011, 74, 1902-1907.

[4]. Dale, J. A.; Mosher, H. S. J. Am. Chem. Soc. 1973, 95, 512-515.

[5]. (a) Ohtani, I.; Kusumi, T.; Ishitsuka, M.O.; Kakisawa, H. Tetrahedron Lett. 1989, 30, 3147-3150. (b) Ohtani, I.; Kusumi, T.; Kashman, Y.; Kakisawa, H. J. Am. Chem. Soc. 1991, 113, $4092-4096$. 